\title{
Prophylactic Probiotics for Prevention of Necrotizing Enterocolitis (NEC) in Low Birth Weight Neonates; Quality of the Evidence
}

\author{
Mario Augusto Rojas ${ }^{1, *}$ \\ ${ }^{1}$ Wake Forest University School of Medicine, Winston-Salem, USA \\ ${ }^{*}$ Corresponding author: Mario Augusto Rojas, Wake Forest University School of Medicine, Winston-Salem, USA. Tel:+1-3367185226, Fax:+1-3367162525, E-mail: mrojas@wakehealth.edu. \\ Received: October 8, 2013; Accepted: October 11, 2013
}

Keywords: Enterocolitis, Necrotizing; Probiotics; Infant, Premature

\section{Dear Editor,}

The study by Dashti et al. is a single center double-blinded placebo controlled trial that exposed infants $\leq 1800$ grams in the intervention group to a multi-species preparation of probiotics. The authors of this paper did not find a difference in the incidence of necrotizing enterocolitis (NEC) between the intervention and the placebo group, nor did they find a difference in any of their secondary outcomes. The paper does not describe the incidence of NEC in their institution and they do not describe how they calculated the sample size based on their historical incidence of NEC. I believe this to be the main weakness of this study and the major reason why the authors of this paper were not powered to find a difference in the primary outcome if in fact this difference did exist. Using the control group incidence of NEC of $1.49 \%$ (stage II and III Bell's classification), a confidence level of $95 \%$, a power of $80 \%$ and an expected decrease of $50 \%$ in the incidence rate of NEC (to $0.75 \%$ ), the total sample size required for this study would have been 6,876 infants $(3,438$ infants in each group). Another possible explanation for the observed lack of efficacy may be due to the inclusion of infants $>1500$ grams, a group that traditionally has a much lower incidence of NEC compared to infants born at $\leq$ 1500 grams. Recruitment of this proportionally large number of more mature infants would tend to dilute the effect of the intervention. Also, the late administration of the probiotic preparation (mean age 4.36 days) when colonization with potential NICU pathogens may already be established could also add to the observed lack of efficacy. The main weaknesses highlighted in this study are not unique to this study but a major theme of the majority of studies included in the multiple meta-analyses published on the use of Probiotics in preterm infants (15). Although these meta-analyses have shown a decrease in the incidence of NEC with the use of different species of probiotics, the potential for publication and selection bias is of great concern because of the known tendency for journals to reject studies that do not show efficacy. From this perspective, I welcome Archives of Pediatric Infectious Diseases's decision to publish this manuscript. Also, the poor quality of the design of many of the studies included in these meta-analyses makes their conclusions at the very least suspicious. Are we ready to start using probiotics for the prevention of NEC in premature infants? Although we understand the urgency of finding an intervention that prevents NEC due to the high mortality and morbidity associated with this outcome, wisdom dictates a more thorough evaluation of the bio-molecular characteristics of specific species, their dosing, quality, and safety. This is especially relevant in the extremely premature infant population where some published studies have demonstrated a higher incidence of sepsis when exposed to probiotics $(6,7)$. I do not believe that we have at the present time a probiotic species that fulfills all these basic requirements. An international consensus agreement that prioritizes and directs future efforts in this area of research is urgently needed to expedite fulfilling these goals. This fragile population of premature infants embraces and supports our coordinated efforts.

\section{Financial Disclosure}

The author has served as a consultant and speaker without honorarium for BioGaia. BioGaia's Lactobacillus reuteri was tested by the author in a randomized controlled trial. BioGaia covered the cost for the probiotic and the placebo in this trial.

\section{References}

1. Barclay AR, Stenson B, Simpson JH, Weaver LT, Wilson DC. Probi-

Copyright (C 2014, Pediartric Infections Research Center. This is an open-access article distributed under the terms of the Creative Commons Attribution License, which permits unrestricted use, distribution, and reproduction in any medium, provided the original work is properly cited. 
otics for necrotizing enterocolitis: a systematic review. J Pediatr Gastroenterol Nutr. 2007;45(5):569-76.

2. Deshpande G, Rao S, Patole S, Bulsara M. Updated meta-analysis of probiotics for preventing necrotizing enterocolitis in preterm neonates. Pediatrics. 2010;125(5):921-30.

3. Alfaleh K, Anabrees J, Bassler D, Al-Kharfi T. Probiotics for prevention of necrotizing enterocolitis in preterm infants. Cochrane Database Syst Rev. 2011(3):CD005496.

4. Wang Q, Dong J, Zhu Y. Probiotic supplement reduces risk of necrotizing enterocolitis and mortality in preterm very low-birthweight infants: an updated meta-analysis of 20 randomized, controlled trials. J Pediatr Surg. 2012;47(1):241-8.
5. Bernardo WM, Aires FT, Carneiro RM, Sa FP, Rullo VE, Burns DA Effectiveness of probiotics in the prophylaxis of necrotizing enterocolitis in preterm neonates: a systematic review and metaanalysis. J Pediatr (Rio J). 2013;89(1):18-24.

6. Dani C, Biadaioli R, Bertini G, Martelli E, Rubaltelli FF. Probiotics feeding in prevention of urinary tract infection, bacterial sepsis and necrotizing enterocolitis in preterm infants. A prospective double-blind study. Biol Neonate. 2002;82(2):103-8.

7. Lin HC, Hsu CH, Chen HL, Chung MY, Hsu JF, Lien RI, et al. Oral probiotics prevent necrotizing enterocolitis in very low birth weight preterm infants: a multicenter, randomized, controlled trial. Pediatrics. 2008;122(4):693-700. 